\title{
Regulation of Biliary Cholesterol Secretion \\ Functional Relationship between the Canalicular and Sinusoidal Cholesterol Secretory Pathways in the Rat
}

\author{
Flavio Nervi, Iván Marinović, Attilio Rigotti, and Natalia Ulloa \\ Departamento de Gastroenterología, Facultad de Medicina, Pontificia Universidad Católica, Santiago, Chile
}

\begin{abstract}
The functional interrelationship between biliary cholesterol secretion, sinusoidal lipoprotein cholesterol secretion and bile salt synthesis was studied in the rat. Diosgenin, fructose, and colestipol in the diet were used to, respectively, influence biliary cholesterol output, VLDL production and bile salt synthesis. In the acute bile fistula rat, biliary cholesterol output was $\mathbf{7 0 0} \%$ increased by diosgenin and $50 \%$ decreased by fructose. In the rats fed both diosgenin and fructose, biliary cholesterol secretion was increased only by $\sim 200 \%$, whereas biliary bile salts and phospholipid outputs were unchanged.

In the isolated perfused liver, VLDL-cholesterol output was $50 \%$ reduced by diosgenin alone, but was unchanged following feeding of diosgenin plus fructose. However, the livers of rats fed diosgenin plus fructose exhibited a $700 \%$ increase in VLDL-triglyceride production and a $200 \%$ increase in VLDLcholesterol output. A significant reciprocal relationship between VLDL-cholesterol secretion and the coupling ratio of cholesterol to bile salts in bile was observed. Colestipol added to the diet maintained both sinusoidal and biliary cholesterol outputs within the normal range.

In the chronic bile fistula rat, colestipol increased bile salt synthesis by $100 \%$ while diosgenin and fructose diets had no effect. Similarly, the addition of fructose to the colestipol diet did not decrease bile salt synthesis.

These data suggest a reciprocal relationship between biliary cholesterol secretion and hepatic secretion of cholesterol as VLDL particles. The free cholesterol pool used for bile salt synthesis seems functionally unrelated to the pool from which VLDL-cholesterol and biliary cholesterol originate. These findings support the idea that metabolic compartmentalization of hepatic cholesterol is a major determinant of the quantity of cholesterol available for recruitment by the bile salt-dependent biliary cholesterol secretory mechanism.
\end{abstract}

\section{Introduction}

The primary pathogenic event in cholesterol gallstone formation is the secretion by the liver of more cholesterol that can be

Presented in part at the IXth International Bile Acid Meeting in Basel, Switzerland, 1986, and at the American Gastroenterological Association Meeting in Chicago, 1987, and published in abstract form (1987. Gastroenterology. 92: 1760a).

Address reprint requests to Dr. Nervi, Pontificia Universidad Católica, Departamento de Gastroenterología, Casilla 114-D, Santiago, Chile.

Received for publication 21 December 1987 and in revised form 29 June 1988.

J. Clin. Invest.

(C) The American Society for Clinical Investigation, Inc.

$0021-9738 / 88 / 12 / 1818 / 08 \quad \$ 2.00$

Volume 82, December 1988, 1818-1825 effectively solubilized by bile salts and phospholipids in bile. This can occur by the secretion of either excessive amounts of cholesterol or decreased amounts of phospholipids or bile salts (1). A significant proportion of patients with cholesterol gallstones, present high rates of biliary cholesterol secretion and normal secretions of bile salts and phospholipids (2-4). These observations suggest that besides the well known effect of bile salts to drive biliary cholesterol output (5-7), there must be other intrahepatic determinants that regulate the amount of cholesterol available for secretion into the bile.

It has been postulated that the rate of hepatic cholesterol synthesis is an important intrahepatic determinant of biliary cholesterol output. This possibility is supported by observations that some patients with cholesterol gallstones have higher activity of the microsomal enzyme 3-hydroxy-3-methylglutaryl coenzyme A reductase compared with subjects without gallstones $(8,9)$, suggesting that hepatic cholesterogenesis is increased in these patients. However other studies both in humans and the rat have not confirmed these observations $(10,11)$, suggesting that the rate of hepatic cholesterogenesis does not directly determine the rate of biliary cholesterol output.

The activity of acyl:CoA cholesterol acyltransferase $(\mathrm{ACAT})^{1}$ (E.C. 2.3.1.26), which esterifies free cholesterol in the liver, may increase or decrease the hepatocyte availability of free cholesterol for either biliary (12-15) or VLDL cholesterol esters secretion (16). Recent studies by us (12-14) and others (15) have indicated that pharmacological manipulation of this enzyme markedly influence biliary cholesterol output. These observations are consistent with the possibility that the major intrahepatic determinant of biliary cholesterol output is the availability of free cholesterol in some hepatic precursor pool whose size would depend on the activity of other metabolic processes, including bile acid synthesis and/or lipoprotein production $(13,14)$. A key aspect of this hypothesis, however, remains unresolved, namely, whether the different excretory pathways of hepatic cholesterol originate in one or more metabolic compartments. Previous studies in man and the rat have suggested that most of biliary cholesterol derives from a preformed pool (17-20), while bile acids, mainly originate from newly synthesized hepatic cholesterol (21-23). The functional interrelationship of these two precursor pools, if any, is presently unknown. Available data suggests that the human hepatocyte preferentially utilizes free cholesterol from HDL for biliary cholesterol secretion (24), but it is uncertain if cholesterol derived from specific lipoproteins or chylomicron remnants may directly drive biliary cholesterol output.

The aim of the present investigation was to ascertain if biliary cholesterol output can be directly influenced by the rates of bile acid synthesis or hepatic lipoprotein production,

1. Abbreviations used in this paper: ACAT, acyl:CoA cholesterol acyltransferase. 
or both. The sinusoidal and biliary secretory pathways of hepatic cholesterol were studied under the dietary manipulations of VLDL production by fructose (25), bile salt synthesis by colestipol (26), and biliary cholesterol output by diosgenin (14).

\section{Methods}

Materials and animals. Cholesterol, diosgenin, triolein, taurocholate, albumin, diosgenin, collagenase, oleic acid, cholesteryl oleate, hydroxysteroid dehydrogenase, NAD and 5,5'-dithiobis (2-nitrobenzoic acid) were purchased from Sigma Chemical Co (St. Louis, MO). Triglyceride enzymatic colorimetric test for automated analysis was purchased from Boehringer Mannheim GmbH (Mannheim, Federal Republic of Germany). 1,2-[ $\left.{ }^{3} \mathrm{H}\right]$ Cholesterol, cholesteryl oleate (1$\left[{ }^{14} \mathrm{C}\right]$ oleate), taurocholic acid $24-\left[{ }^{14} \mathrm{C}\right]$, and $1-\left[{ }^{14} \mathrm{C}\right]$ oleic acid were obtained from New England Nuclear (Boston, MA). Silicic acid was obtained from Mallinckrodt Chemical Works (St. Louis, MO). Celite was obtained from Johns-Manville Products Corp. (Denver, CO). Fructose, silica gel, and all organic solvents were obtained from E. Merck (Darmstadt, Federal Republic of Germany). Polyethylene catheters were purchased from Clay Adams Inc. (Parsippany, NJ) and silastic tubes from Dow Corning Co (Midland, MI).

Male Wistar rats in the weight range $80-120 \mathrm{~g}$ were subjected to reversed light cycling for 2-3 wk before use. The mid-dark point was set at 10 a.m. The animals were fed ground chow that contained (wt/vol) cholesterol 0.013-0.02\%. Some groups received ground chow that contained (wt/vol) $1 \%$ diosgenin, $30 \%$ fructose, $5 \%$ colestipol, $1 \%$ diosgenin plus $30 \%$ fructose or $5 \%$ colestipol plus $30 \%$ fructose for 7 to $9 \mathrm{~d}$ before the experiments. Fructose and colestipol were directly mixed with the chow, whereas diosgenin was previously dissolved in chloroform, mixed with the diets, and the solvent evaporated by heating $24 \mathrm{~h}$ at $37^{\circ} \mathrm{C}$ under a hood.

Biliary secretion studies in vivo. Bile was collected in a preweighed tube for 30-min periods. Bile specimens were obtained during the first 30 min of bile fistula (initial bile specimens), during depletion of the bile salt pool or intravenous taurocholate infusion in specific experiments, as previously described (12). In experiments designed to study the effect of experimental diets on the relationship between bile salts secretion and cholesterol or phospholipids secretion, the data was treated as previously described $(7,12)$. Briefly, simulated curves were derived with the experimental data by standard computer procedures (B.M.D. PAR Subroutine, Biomedical Computer Programs P-Series, University of California, Los Angeles, CA). As expected the best curve fitting for the experimental data was a nonlinear regression of the form $y=a x / b x$, where a represents the maximal theoretical output of biliary lipid obtained after a specific dietary manipulation.

A chronic bile fistula animal was prepared to estimate total bile salt synthesis at the nadir of bile salt secretion as previously described (27). $12-15 \mathrm{~h}$ before the experiment the animals received an intraperitoneal dose of $0.4 \mu \mathrm{Ci}$ taurocholic acid $24-{ }^{14} \mathrm{C}$. The initial $10 \mathrm{~min}$ of bile specimen was used to calculate the specific activity of the bile salt pool at start of the bile salt pool depletion period. After $12 \mathrm{~h}$ of bile collection $<1 \%$ of total bile salts output were originated from the initial bile salt pool. Therefore, bile salt secreted after $12 \mathrm{~h}$ of bile fistula was considered newly synthetized bile salts. In preliminary experiments we found that derepression of bile salt synthesis occurred after $28 \mathrm{~h}$ of bile fistula. Bile salt synthesis was estimated averaging bile salt output values of three 1-h bile specimens collected after 15-18 h of bile fistula, which occurred during the mid-dark phase of the diurnal cycle 8 to 12 a.m. Bile salt synthesis was expressed as nanomoles per hour per gram of liver weight.

Liver perfusion. On the day of the experiments, the rats were anesthetized with an intraperitoneal injection of Nembutal $(4.5 \mathrm{mg} / 100 \mathrm{~g}$ body wt) at 9-10:30 a.m. The bile duct and portal vein were cannulated with PE 10 and PE indwelling Braunula, respectively. The inferior vena cava was then ligated after sectioning the superior vena cava in the thoracic cavity. During surgery and transfer to the perfusion apparatus, the liver was continuously perfused with Krebs-Henseleit buffer (28) containing $0.1 \%\left(\mathrm{wt} / \mathrm{vol}\right.$ ) glucose and maintained at $37^{\circ} \mathrm{C}$ while oxygenated with $95 \% \mathrm{O}_{2}$ and $5 \% \mathrm{CO}_{2}$. The liver was then perfused with 70-80 ml of recirculating Krebs-Henseleit medium, which in addition contained $0.1 \%(\mathrm{wt} / \mathrm{vol})$ glucose and $2 \%(\mathrm{wt} / \mathrm{vol})$ fatty acid free bovine albumin at a rate of $20 \mathrm{ml} / \mathrm{min}$ at $37^{\circ} \mathrm{C}$. Taurocholate was continuously infused into the perfusate at a rate of $0.45 \mu \mathrm{mol} / \mathrm{min}$. The perfusate was oxygenated with $95 \% \mathrm{O}_{2}$ and $5 \% \mathrm{CO}_{2}$ using the apparatus described by Hamilton et al. (29). Perfusion was maintained for $90 \mathrm{~min}$. Bile was collected at 30-min intervals and in some experiments, a $12-15-\mathrm{ml}$ aliquot of the perfusate was obtained after $40 \mathrm{~min}$ of perfusion to measure lipoprotein triglyceride and cholesterol outputs. Liver viability was assessed by bile flow, bile salt secretion, oxygen extraction, and gross appearance.

Isolation of perfusate lipoproteins. At the end of the perfusion period, $0.05 \%$ EDTA and $0.05 \%$ 5,5'-dithiobis (2-nitrobenzoic acid) were added to the perfusates. For the isolation of VLDL $\left(d<1.006 \mathrm{~g} \times \mathrm{ml}^{-1}\right.$ fractions) samples of perfusates were centrifuged at $105,000 \mathrm{~g}$ and $4^{\circ} \mathrm{C}$ for $22 \mathrm{~h}$. The VLDL fraction was recovered quantitatively in the top $3.5 \mathrm{ml}$ of the $9.5-\mathrm{ml}$ capacity centrifuge tubes.

Determination of hepatic cholesterol esterification in isolated hepatocytes. Hepatic cholesterol ester synthesis was measured as previously described (12). Briefly, isolated hepatocytes from fed rats were prepared as described by Berry and Friend (30). The incubations were started by addition of $0.2 \mathrm{ml}$ of cell suspension (7-10 $\mathrm{mg}$ of protein) to $0.8 \mathrm{ml}$ of buffer that contained $1 \mathrm{mM} 1-\left[{ }^{14} \mathrm{C}\right]$ oleic acid $(0.70 \mu \mathrm{Ci} / \mathrm{ml})$. The incubation time was $10 \mathrm{~min}$. Incubation were performed in $25 \mathrm{ml}$ erlenmeyer flasks oxygenated with $5 \% \mathrm{CO}_{2}$ in $95 \% \mathrm{O}_{2}$ at $37^{\circ} \mathrm{C}$.

After extraction with $20 \mathrm{vol}$ chloroform-methanol 2:1, cholesterol oleate was separated using a solvent mixture of hexane ethyl-acetate 9:1. Cholesterol ester was located by comparison with cholesterol oleate standard. The spot corresponding to cholesterol ester was scraped from the plates into counting vials for radioactivity determination.

Analytical methods. Free and esterified cholesterol were extracted and separated as previously described $(13,31)$. Cholesterol was quantitated by the colorimetric method of Zak (32). Phospholipids were measured in the chloroform phase of chloroform-methanol extracts by the colorimetric method of Baginski et al. (33). Bile salts were quantitated by the $3 \alpha$-hydroxysteroid dehydrogenase method of Talalay (34) modified by Turley and Dietschy (35). Lipoprotein triglycerides were measured by the Boehringer Mannheim Automated Analysis for BM/ Hitachi System 704. Hepatocyte protein was measured by the method of Lowry et al. (36).

Statistics. Results are presented as the mean \pm SE. Differences in mean values were tested for significance using the unpaired $t$ test (37). A difference was considered significant with $P$ values $\leq 0.05$.

\section{Results}

The effect of fructose, diosgenin and colestipol feeding on bile secretion and biliary cholesterol saturation in vivo. The first series of experiments was undertaken to characterize bile secretion in the different groups of rats subjected to the experimental diets. The diets were well accepted by the animals. The increase in body weight during the 7 to $9 \mathrm{~d}$ of experimental diets were similar in all groups. The final body weight at the moment of the experiments were in normal ranges, in rats fed either fructose, diosgenin or colestipol as shown in Table I. Bile flow significantly decreased by $28 \%$ in the colestipol-fed rats probably as a result of the significant $33 \%$ decrease of biliary bile salt output. In contrast, neither fructose nor diosgenin modified these parameters. Biliary phospholipid output remained in the normal range in the different groups of animals. However, biliary cholesterol output significantly de- 


\begin{tabular}{|c|c|c|c|c|c|c|c|}
\hline \multirow[b]{2}{*}{ Group } & \multirow[b]{2}{*}{ Body wt } & \multirow[b]{2}{*}{ Liver wt } & \multirow[b]{2}{*}{ Bile flow } & \multicolumn{3}{|c|}{ Biliary lipid output } & \multirow{2}{*}{$\begin{array}{l}\text { Biliary cholesterol } \\
\text { molar percentage }\end{array}$} \\
\hline & & & & Bile salt & Phospholipid & Cholesterol & \\
\hline & $g$ & $g$ & $\mu l \times g^{-1} \times \min ^{-1}$ & \multicolumn{3}{|c|}{$n \mathrm{~mol} \times \mathrm{g}^{-1} \times \mathrm{min}^{-1}$} & \\
\hline A Control & $188 \pm 7$ & $7.3 \pm 0.6$ & $2.1 \pm 0.1$ & $90 \pm 8$ & $11 \pm 1.5$ & $1.2 \pm 0.2$ & $1.2 \pm 0.1$ \\
\hline B Fructose $30 \%$ & $195 \pm 4$ & $7.0 \pm 0.3$ & $2.2 \pm 0.3$ & $114 \pm 14$ & $9 \pm 1.2$ & $0.5 \pm 0.1^{*}$ & $0.4 \pm 0.03^{*}$ \\
\hline C Dịgsenin $1 \%$ & $201 \pm 9$ & $7.9 \pm 0.8$ & $2.4 \pm 0.3$ & $87 \pm 5$ & $14 \pm 1.0$ & $9.9 \pm 2.7^{*}$ & $8.9 \pm 0.5^{*}$ \\
\hline D Colestipol 5\% & $183 \pm 13$ & $6.7 \pm 0.6$ & $1.5 \pm 0.2^{*}$ & $60 \pm 4^{*}$ & $9 \pm 0.4$ & $1.0 \pm 0.3$ & $1.4 \pm 0.1$ \\
\hline E Fructose $30 \%+$ diosgenin $1 \%$ & $198 \pm 9$ & $7.7 \pm 1.1$ & $2.1 \pm 0.3$ & $88 \pm 9$ & $13 \pm 1.3$ & $2.8 \pm 0.6^{*}$ & $3.1 \pm 0.6^{*}$ \\
\hline F Fructose $30 \%+$ colestipol $5 \%$ & $180 \pm 7$ & $7.8 \pm 0.6$ & $1.7 \pm 0.1$ & $60 \pm 7$ & $9 \pm 0.5$ & $0.3 \pm 0.1^{*}$ & $0.4 \pm 0.02 *$ \\
\hline
\end{tabular}

All groups were fed ground chow for 7 to $9 \mathrm{~d}$ before the experiment. There were five to seven animals in each group. Values are the mean \pm 1 SE. The asterisks shown in groups B, C, and D indicate that the value is significantly different at the $P<0.05$ level from the A group. The asterisks shown in group $\mathrm{E}$ indicate that the values are significantly different from group $\mathrm{C}$ and those from group $\mathrm{F}$ are significantly different from group $\mathrm{D}$ at the $P<0.05$ level.

creased by almost $60 \%$ in fructose-fed rats and significantly increased by $700 \%$ over the control value in diosgenin fed rats. This parameter remained in the normal range in the colestipol-fed rats (group $D$, Table I). These modifications of biliary cholesterol output were paralleled by changes of biliary cholesterol molar percentage in the same direction. When $30 \%$ fructose was added to the $1 \%$ diosgenin diet biliary cholesterol output and saturation decreased by 72 and $65 \%$, respectively, compared to the $1 \%$ diosgenin-fed animals (groups $E$ vs. $C$, Table I).

The effects of fructose on the changes induced by colestipol and diosgenin on biliary lipid outputs were next studied. As shown in Table I, it was found that the addition of fructose to the colestipol diet did not modify biliary bile salt output (Table I, group $F$ vs. $D$ ), whereas biliary cholesterol output significantly decreased by $70 \%$ (Table I, group $F$ vs. $D$ ). This change of biliary cholesterol output was similar to that found in control animals fed $30 \%$ fructose (Table I, group $B$ vs. $A$ ). The kinetic characteristics of the relationship between biliary cholesterol and phospholipid outputs as a function of biliary bile salt output in diosgenin-fed and diosgenin plus fructose-fed animals is shown in Fig. 1. The secretory relationship between biliary phospholipid and bile salt outputs were similar in both groups of rats (Fig. $1 \mathrm{~B}$ ). In contrast, it was apparent that the amount of biliary cholesterol secreted per unit of bile salt was considerably lower in the diosgenin plus fructose-fed animals (Fig. $1 \mathrm{~A}$ ). The calculated maximal cholesterol output of this group decreased from $14 \pm 2$ to $3 \pm 0.4(P<0.05) \mathrm{nmol} \times \mathrm{g}^{-1}$ $X$ min $^{-1}$. It is important to note that under these experimental conditions, the maximal theoretical cholesterol output of our male Wistar rats (17) is in the range of that found in the fructose-fed animals.

As a result of these experiments it was concluded that the stimulation of VLDL production by fructose markedly decreased biliary cholesterol output. This finding was found whether the animals were fed with colestipol to stimulate bile salt synthesis, or diosgenin to stimulate biliary cholesterol output.

The effects of fructose, diosgenin and colestipol feeding on lipoprotein triglyceride and cholesterol outputs in vitro. The rate of bile flow and biliary bile salt output by the perfused rat liver remained constant during the entire $90-\mathrm{min}$ period of these studies. As shown in Table IV, the average bile flow rate varied between 90 and $117 \mu \mathrm{l} \times \mathrm{g}^{-1} \times 90 \mathrm{~min}^{-1}$ in the control, diosgenin-fed, and diosgenin plus fructose-fed rats, which was in the range reported by others $(38-41)$. Each 30 -min period of bile flow represented one third of the total volume of bile produced. Similarly, bile salt output was similar in the three experimental groups. Each 30 -min period of bile salt output

(A)
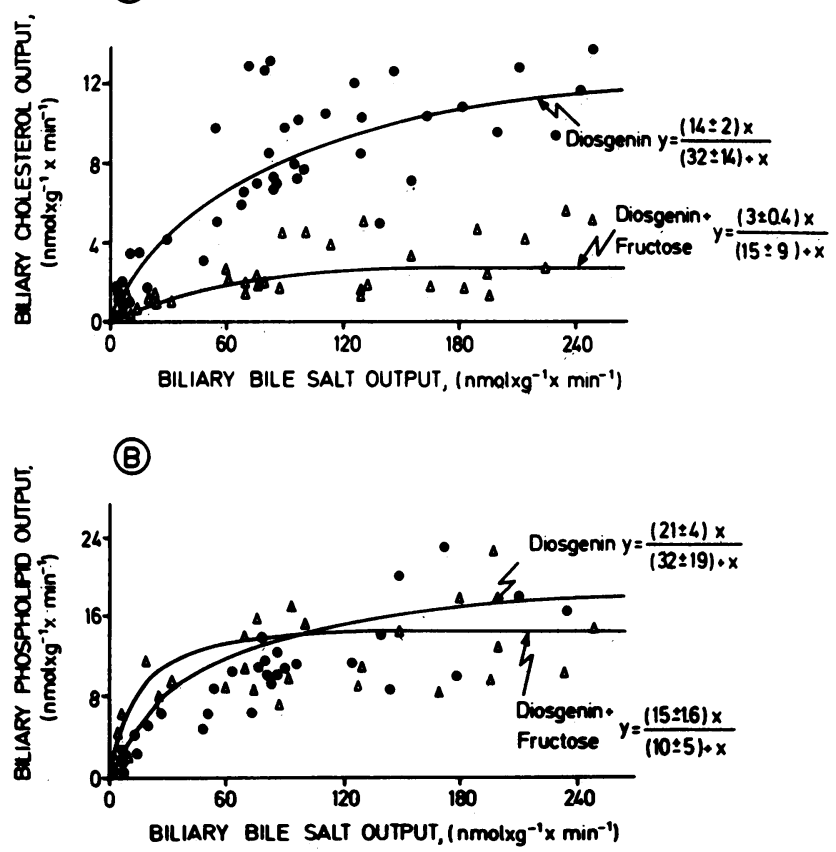

Figure 1. Relationship between the rates of biliary cholesterol $(A)$ and phospholipid outputs $(B)$, and biliary bile salt output. The curves were derived from data obtained from $1 \%$ diosgenin-fed and $30 \%$ fructose $+1 \%$ diosgenin-fed animals. Each group was formed with the rats of Table I, two rats being infused with $45 \mathrm{mM}$ taurocholate in $0.15 \mathrm{M} \mathrm{NaCl}$ at a rate of $0.075 \mathrm{ml} \times \mathrm{min}^{-1}$ for a period of 40 min (equivalent to two or three bile samples), a third group of two rats being subjected to acute depletion of the bile salt pool. Four to six bile specimens were obtained from each of these animals: curve fitting of the data is represented by the rectangular hyperbola $y$ $=a x / b+x$. The theoretical maximal cholesterol output of the fructose + diosgenin-fed rats significantly decreased by $>400 \%(P$ $<0.001)$. 
represented $30-33 \%$ of the total bile salt output collected during the 90 -min experimental period.

Control livers secreted triglyceride actively at the beginning of the perfusion, although net triglyceride output subsequently declined (Fig. $2 A$ ). Diosgenin did not change triglyceride output, but the addition of $30 \%$ fructose to this diet markedly stimulated triglyceride secretion by $700 \%$ in the VLDL fraction. A minor, but significant increment, was also found in the $d>1.006 \mathrm{~g} \times \mathrm{ml}^{-1}$ lipoprotein fraction. Colestipol also slightly increased VLDL triglyceride output after $90 \mathrm{~min}$ of perfusion by $\sim 70 \%$. This observation is consistent with previous studies in which colestipol increased the plasma level and hepatic syn-
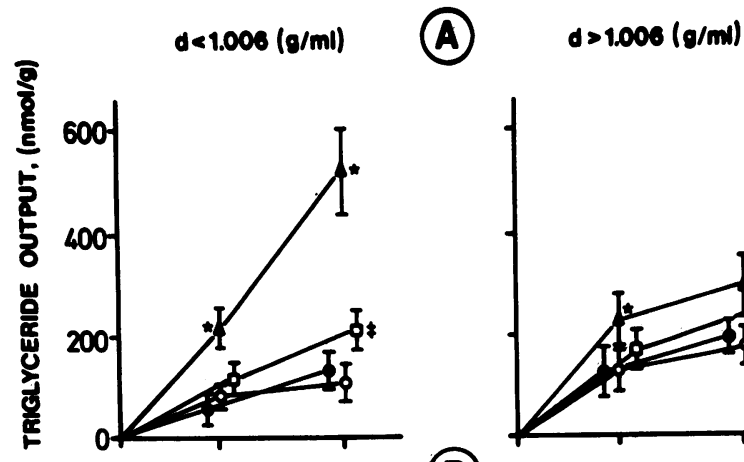

(B)
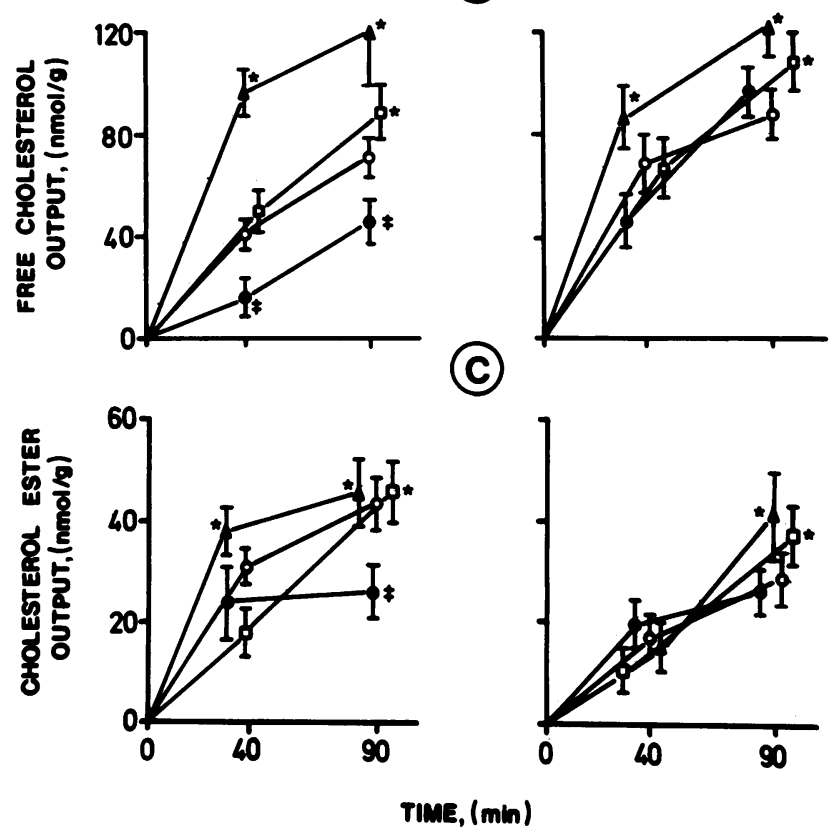

Figure 2. Effect of diosgenin, fructose, and colestipol on the net secretion of triglyceride and cholesterol in the $d<1.006 \mathrm{~g}^{-1}$ (VLDL) and $d>1.006 \mathrm{~g}^{-1}$ fractions by the perfused liver. Livers from fed rats were perfused with Krebs-Henseleit medium containing $0.1 \%$ glucose. Taurocholate $\left(0.45 \mu \mathrm{mol} \times \mathrm{min}^{-1}\right)$ was continuously infused into the perfusates throughout the experiments. Each point represents the mean $\pm \mathrm{SE}$ of seven control, seven $1 \%$ diosgenin-fed rats, five $30 \%$ fructose $+1 \%$ diosgenin-fed, and five colestipol-fed animals. A, B, and C represent lipoprotein triglyceride, free cholesterol, and cholesterol esters outputs, respectively. $\phi$, control; $\phi$, diosgenin; $\phi$, colestipol; 4 , fructose + diosgenin. The asterisk indicates a significant difference at the $P<0.05$ level (compared to control and diosgenin-fed rats). The double cross indicates a significant difference at the $P<0.05$ level (compared to control rats). thesis of triacylglycerol, indicating an enhanced VLDL production (26).

Lipoprotein free cholesterol secretion is shown in Fig. $2 B$. Diosgenin-fed rats significantly decreased free cholesterol output by more than $50 \%$ in the $d<1.006 \mathrm{~g} \times \mathrm{ml}^{-1}$ lipoprotein density fraction (VLDL); the addition of fructose to the diosgenin diet significantly increased free cholesterol output by more than $200 \%$ in VLDL. In contrast, colestipol did not modify lipoprotein free cholesterol output. This parameter remained in the normal range in the $d>1.006 \mathrm{~g} \times \mathrm{ml}^{-1}$ fraction in diosgenin-fed rats and slightly, but significantly increased, in the diosgenin plus fructose-fed animals. The effect of the different experimental diets on lipoprotein cholesterol esters output is shown in Fig. $2 C$. Cholesterol ester output remained in the control level in the diosgenin-fed rats during the first $\mathbf{4 0}$ min of perfusion and remained in the same level at $90 \mathrm{~min}$ of perfusion. VLDL cholesterol ester output was significantly decreased by $45 \%$ in the diosgenin-fed rats. The addition of fructose to the diosgenin diet restored VLDL cholesterol ester secretion to the control level. The different experimental diets did not modify this parameter in the $d>1.006 \mathrm{~g} \times \mathrm{ml}^{-1}$ lipoprotein fraction; with the exception of fructose, which significantly increased cholesterol ester output by $77 \%$ compared with the diosgenin diet.

The relative amounts of VLDL triglyceride to free cholesterol and cholesterol esters to total cholesterol were changed by some of the experimental diets as shown in Table II. The ratios of triglyceride to free cholesterol of control animals were $\sim 2$, which is similar to the ratios found in previous studies (40). Colestipol-fed rats secreted VLDL particles with the same characteristics; whereas diosgenin-fed animals secreted VLDL particles with a higher proportion of triglyceride as a consequence of the lower free cholesterol outputs. The ratios of cholesterol esters to total cholesterol in the diosgenin plus fructose-fed rats were significantly lower as compared with the diosgenin-fed rats.

The effect of diosgenin on the accumulation of cholesterol in three different liver perfusate lipoprotein density fractions is shown in Table III. Approximately $50 \%$ of cholesterol of the $d$ $>1.006$ fraction is found in a density fraction $1.006<d$ $<1.063 \mathrm{~g} \times \mathrm{ml}^{-1}$ that corresponds with LDL. This fraction has been considered as functionally similar to VLDL when found

Table II. Relative Triglyceride and Cholesterol Composition of Liver Perfusate $d<1.006 \mathrm{~g} \times \mathrm{ml}^{-1}$ Lipoproteins

\begin{tabular}{|c|c|c|c|c|}
\hline \multirow[t]{2}{*}{ Group } & \multicolumn{2}{|c|}{$\begin{array}{l}\text { Ratios of triglyceride to } \\
\text { free cholesterol }\end{array}$} & \multicolumn{2}{|c|}{$\begin{array}{l}\text { Ratios of cholesterol esters to } \\
\text { total cholesterol }\end{array}$} \\
\hline & $40 \mathrm{~min}$ & $90 \min$ & $40 \mathrm{~min}$ & $90 \mathrm{~min}$ \\
\hline A Control & $2: 4 \pm 0.8$ & $1.6 \pm 0.4$ & $0.47 \pm 0.03$ & $0.36 \pm 0.03$ \\
\hline B Diosgenin $1 \%$ & $4.6 \pm 0.8^{*}$ & $3.4 \pm 0.9^{*}$ & $0.50 \pm 0.06$ & $0.33 \pm 0.03$ \\
\hline C Colestipol 5\% & $2.0 \pm 0.4$ & $2.4 \pm 0.6$ & $0.38 \pm 0.08$ & $0.30 \pm 0.04$ \\
\hline \multicolumn{5}{|l|}{ D Diosgenin $1 \%$} \\
\hline+ fructose $30 \%$ & $2.7 \pm 0.5^{*}$ & $4.5 \pm 0.7$ & $0.29 \pm 0.04^{*}$ & $0.25 \pm 0.02^{*}$ \\
\hline
\end{tabular}

The ratios were calculated from the composition of the lipoprotein fractions separated in the experiments shown in Fig. 2.

* The values are significantly different from group $A$ and those shown in group D are significantly different from group B at the $P$ $<0.05$ level. 
Table III. Effect of Diosgenin on Accumulation of Cholesterol in Liver Perfusate Lipoprotein Density Fractions

\begin{tabular}{|c|c|c|}
\hline \multirow[b]{2}{*}{ Lipoprotein density fraction } & \multicolumn{2}{|c|}{ Total cholesterol } \\
\hline & Control (4) & Diosgenin (3) \\
\hline$g \times m l^{-1}$ & \multicolumn{2}{|c|}{$n m o l \times m l^{-1}$} \\
\hline$d<1.006$ & $10.6 \pm 1.5$ & $7.4 \pm 1.4$ \\
\hline $1.006<d<1.063$ & $7.1 \pm 0.4$ & $4.7 \pm 1.0$ \\
\hline$d>1.063$ & $6.1 \pm 1.0$ & $5.1 \pm 0.7$ \\
\hline
\end{tabular}

Livers were perfused with a continuous infusion of taurocholate $\mathbf{0 . 4 5}$ $\mu \mathrm{mol} \times \min ^{-1} \times 90 \mathrm{~min}$

Perfusates were collected and separated into three density fractions by sequential ultracentrifugation after adjusting the densities with $\mathrm{KBr}$. The amount of cholesterol in each fraction was determined and expressed as nanomoles per milliliter of perfusate fractionated after $90 \mathrm{~min}$ of perfusion. Figures in parentheses represent the number of perfusions. Values represent the mean $\pm \mathrm{SE}$.

in liver perfusate lipoprotein density fractions (42). This finding may explain the significant increment in triglyceride secretion induced by fructose in the $d>1.006 \mathrm{~g} \times \mathrm{ml}^{-1}$ fraction (Fig. 2).

The interrelationship between biliary cholesterol and lipoprotein cholesterol was studied in a series of isolated perfused livers from control, diosgenin and diosgenin plus fructose-fed animals as shown in Table IV and Fig. 3. It can be seen that both bile flow and biliary bile salt output were maintained constant in the three groups of livers. It is important to note that the absolute rates of biliary cholesterol outputs found in the isolated perfused livers are in the range found in vivo. Biliary cholesterol output in isolated control livers was equivalent to $1.55 \mathrm{nmol} \times \mathrm{g}^{-1} \times \mathrm{min}^{-1}$, which is comparable to the

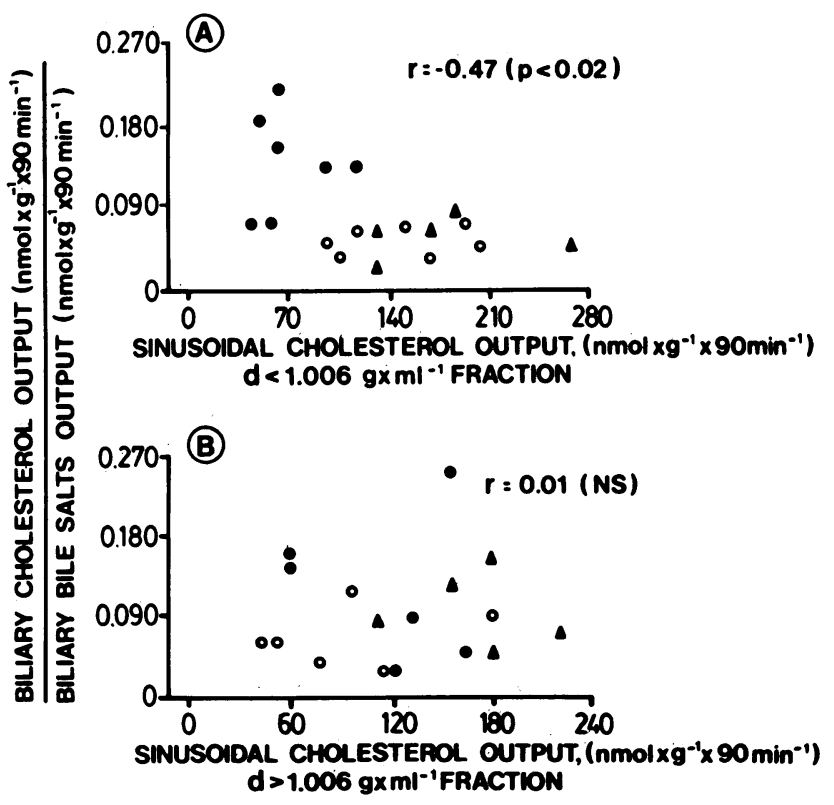

Figure 3. Relationship between the ratio biliary cholesterol to biliary bile salt outputs and sinusoidal cholesterol output in $d<1.006 \mathrm{~g}$ $\times \mathrm{ml}^{-1}$ (VLDL), $(A)$ and in $d>1.006 \mathrm{~g} \times \mathrm{ml}^{-1}$ fractions. $\phi$, control; $\phi$, diosgenin; 4 , fructose + diosgenin. rate found in vivo, as shown in Table I. The ratio biliary cholesterol output to bile salts output was significantly correlated to sinusoidal cholesterol output of $d<1.006 \mathrm{~g} \times \mathrm{ml}^{-1}$ (VLDL) fraction in a reciprocal manner. In contrast, no correlation was found between the ratio biliary cholesterol output to bile salts output with sinusoidal cholesterol output of $d$ $>1.006 \mathrm{~g} \times \mathrm{ml}^{-1}$.

The effect of diosgenin and fructose on serum and hepatic cholesterol, and on $\left[{ }^{14} \mathrm{C}\right]$ oleate incorporation into hepatic cholesterol esters. As shown in Table $\mathrm{V}$, neither diosgenin nor fructose modified serum cholesterol or hepatic cholesterol concentration, free and ester fractions. However, oleate incorporation into cholesterol esters were significantly inhibited in both diosgenin and diosgenin plus fructose-fed rats by $35 \%$ and $55 \%$, compared to control and fructose-fed animals, respectively. These experiments confirmed the inhibitory effect of diosgenin on hepatic cholesterol ester synthesis previously described (14), a result that correlates with the lower rates of VLDL cholesterol ester output found in these animals (Fig. 2). The fact that the addition of fructose to the diosgenin diet normalized VLDL cholesterol ester output (Fig. 2), but did not increase $\left[{ }^{14} \mathrm{C}\right]$ oleate incorporation into cholesterol esters in isolated hepatocytes presumably indicates a mobilization of a higher proportion of the intrahepatic cholesterol esters that are accessible to the mechanism of VLDL synthesis. This mechanism is known to be stimulated by fructose (25).

The effect of diosgenin, colestipol and fructose on bile salt synthesis. An estimate of the effect of the different experimental diets on total bile salt synthesis was obtained with the chronic bile fistula animal as shown in Fig. 4. It is important to note that only colestipol-fed rats significantly increased bile salt synthesis by $\sim 100 \%$, whereas no change was observed in the diosgenin-fed group of rats. The addition of fructose to the colestipol diet to increase VLDL output did not reverse the increment of bile salt synthesis induced by colestipol.

\section{Discussion}

The present study indicates that fructose feeding decreases biliary cholesterol output and simultaneously stimulates VLDL triglyceride and cholesterol outputs in the rat. In addition, fructose blocks the stimulatory effect of diosgenin on biliary cholesterol output, suggesting the presence of a controlling mechanism of biliary cholesterol output, which depends on VLDL assembly and secretion. In contrast, colestipol, which stimulates bile salt synthesis, did not affect canalicular and sinusoidal lipoprotein cholesterol outputs, a finding compatible with the possibility that the cholesterol precursor pool for bile salt synthesis is not functionally connected with the precursor pool(s) for lipoprotein and biliary cholesterol outputs. A summary indicating the effects of the different dietary manipulations used in this study on hepatic cholesterol secretion is shown in Fig. 5.

Approximately $80 \%$ of hepatocytic cholesterol is in the plasma membrane as a structural constituent (43), while up $20 \%$ of total hepatic cholesterol is located in intracellular membranes, mainly in the endoplasmic reticulum and Golgi. These organelles represent the metabolic compartments where VLDL components are assembled (40). There is substantial evidence that biliary lipids also originate in the endoplasmic reticulum $(41,44)$. Metabolically active hepatic cholesterol originates from both new synthesis, and from chylomicron or 
Table IV. Effect of Fructose and Diosgenin on Biliary and Sinusoidal Cholesterol Output In Vitro

\begin{tabular}{|c|c|c|c|c|c|c|}
\hline & Bile flow & Biliary bile salt output & Biliary cholesterol output & $\begin{array}{l}\text { Ratios of biliary } \\
\text { cholesterol to bile salts }\end{array}$ & \multicolumn{2}{|c|}{$\begin{array}{l}\text { Lipoprotein total cholesterol } \\
\left(\mu \mathrm{mol} \times \mathrm{g}^{-1} \times 90 \mathrm{~min}^{-1}\right)\end{array}$} \\
\hline & \multicolumn{3}{|c|}{$\mu l \times g^{-1} \times 90 \mathrm{~min}^{-1}$} & & \multicolumn{2}{|c|}{$d<1.006 \mathrm{~g} \times \mathrm{ml}^{-1}$} \\
\hline A Control (7) & $90 \pm 6.3$ & $4.0 \pm 0.4$ & $0.14 \pm 0.03$ & $0.035 \pm 0.007$ & $0.130 \pm 0.02$ & $0.081 \pm 0.017$ \\
\hline B Diosgenin $1 \%(7)$ & $90 \pm 8.1$ & $3.6 \pm 0.4$ & $0.43 \pm 0.06^{*}$ & $0.131 \pm 0.03^{*}$ & $0.071 \pm 0.01^{*}$ & $0.114 \pm 0.02$ \\
\hline C Diosgenin $1 \%+$ fructose $30 \%(5)$ & $117 \pm 13$ & $3.9 \pm 0.4$ & $0.19 \pm 0.04^{*}$ & $0.052 \pm 0.008^{*}$ & $0.161 \pm 0.03^{*}$ & $0.184 \pm 0.01^{*}$ \\
\hline
\end{tabular}

Livers were perfused with a continuous infusion of taurocholate $0.45 \mu \mathrm{mol} \times \mathrm{min}^{-1}$. The number of perfusions in each group is shown in parentheses. Values represent the mean $\pm \mathrm{SE}$. ${ }^{*}$ The value is significantly different at the $P<0.05$ level: B vs. A; C vs. B.

VLDL remnants, LDL and HDL. The last two lipoproteins deliver cholesterol into the hepatocyte through either a receptor- or a nonreceptor-mediated pathway. The influx of cholesterol from these pathways would supply the cellular needs for membrane new synthesis, lipoprotein production, bile acid synthesis, and biliary cholesterol secretion. In a situation of relative cellular excess, free cholesterol is immediately esterified by ACAT for cholesterol storage or reexcretion in VLDL particles (45).

A similar situation will occur during inhibition of long chain fatty acid oxidation (40). The extra cholesterol required for VLDL synthesis leads to a major decrease in biliary cholesterol output, as exhibited by the fructose-fed animals (Table I). The higher demand for more VLDL free cholesterol is met under both basal conditions (control diet), or under a high biliary cholesterol secretory state, as it occurs with diosgenin feeding. The physiologic implication of this finding is that both secretory pathways of hepatic cholesterol are functionally interrelated and that the system for VLDL assembly may modulate the amount of free cholesterol available for recruitment by bile salts and secretion into the bile. Thus, when VLDL-triglyceride secretion is relatively low, i.e., when animals are fed the control diet, and the biliary cholesterol output is driven by diosgenin, VLDL cholesterol, free and ester fractions, will slightly decrease, but will be sufficient to maintain a normal rate of VLDL-triglyceride output. Thus, metabolic and hormonal conditions that modulate hepatic VLDL-triglyceride output may also constitute important intrahepatic determinants of biliary cholesterol output.

Previous studies from our laboratory (14) strongly support the concept that VLDL and biliary lipid newly synthesized particles originate from the same endoplasmic reticulum precursor pool. Recently, biliary lipid vesicles have been found in unsaturated rat bile, suggesting that hepatocytes normally secrete biliary lipids as bilamellar vesicles into the canalicular lumen (46). Although the intracellular pathways for VLDL transport from the rough endoplasmic reticulum into the plasma membrane have been characterized $(47,48)$, there is no information on the possible intracellular pathway followed by this precursor of biliary vesicles. The finding that colchicine inhibits biliary phospholipid and cholesterol outputs (49), strongly supports the possibility that biliary vesicles are transported from their site of assembly in the endoplasmic reticulum to the canalicular membrane through the microtubular system. In this model, hepatic free cholesterol secretion into bile would depend on a balance between the quantity and species of bile salts interacting with the endoplasmic reticulum (to vectorially drive phospholipid-cholesterol vesicles into the canaliculi), ACAT activity, and the rate of VLDL synthesis and secretion through the sinusoidal membrane. It is apparent from the experiments shown in Fig. 2, and Table IV, that besides the previously shown functional relationship between the activity of the ACAT reaction and biliary cholesterol output (12-14), there is also a reciprocal relationship between this

Table V. The Effect of Diosgenin and Fructose on Serum and Hepatic Cholesterol and on $\left[{ }^{14} C\right]$ Oleate Incorporation into Hepatic Cholesterol Esters

\begin{tabular}{|c|c|c|c|c|}
\hline \multirow[b]{2}{*}{ Group } & \multirow[b]{2}{*}{ Serum cholesterol } & \multicolumn{2}{|c|}{ Hepatic cholesterol } & \multirow{2}{*}{$\begin{array}{l}{\left[{ }^{14} \mathrm{C}\right] \text { Oleate incorporation }} \\
\text { into cholesterol esters }\end{array}$} \\
\hline & & Free & Ester & \\
\hline & $\mu m o l \times d l^{-1}$ & \multicolumn{2}{|c|}{$\mu \mathrm{mol} \times \mathrm{g}^{-1}$} & $p m o l \times m g^{-1} \times h^{-1}$ \\
\hline A Control (6) & $189 \pm 8$ & $4.9 \pm 0.15$ & $0.72 \pm 0.08$ & $33.5 \pm 5.7$ \\
\hline B Diosgenin $1 \%(4)$ & $168 \pm 22$ & $5.4 \pm 0.6$ & $0.75 \pm 0.13$ & $21.7 \pm 4.7^{*}$ \\
\hline C Fructose $30 \%(3)$ & $183 \pm 20$ & $5.2 \pm 0.6$ & $0.72 \pm 0.17$ & $41.8 \pm 4.6$ \\
\hline D Diosgenin $1 \%+$ fructose $30 \%(4)$ & $170 \pm 8$ & $5.1 \pm 0.5$ & $0.65 \pm 0.16$ & $18.7 \pm 4.0^{*}$ \\
\hline
\end{tabular}

Rates of oleate incorporation into cholesterol esters were determined in isolated hepatocytes at the mid-dark point of the diurnal cycle. The cells were resuspended in Krebs-Henseleit buffer, $\mathrm{pH} 7.4$, containing $2.5 \mathrm{mM} \mathrm{CaCl}_{2}$. Each incubation flask contained $0.2 \mathrm{ml}$ of cell suspension, $0.5 \mathrm{mM}$ bovine albumin, and $1 \mathrm{mM}\left[1-\left[{ }^{14} \mathrm{C}\right)\right.$ oleic acid in a total volume of $1 \mathrm{ml}$. Flasks were incubated at $37^{\circ} \mathrm{C}$ for $10 \mathrm{~min}$ in an atmosphere of $95 \% \mathrm{O}_{2}$ and $5 \% \mathrm{CO}_{2}$. The reaction was stopped with $19 \mathrm{ml}$ of chloroform-methanol (2:1). Results of incorporation are expressed as the picomole incorporated into product per milligram of protein per hour. All determinations were performed in triplicate. Hepatic cholesterol concentrations are expressed as $\mu \mathrm{mol}$ per gram of liver weight. ${ }^{*}$ Significant difference at the $P<0.05$ level (B vs. A; D vs. C). The number of rats in each group are shown in parentheses. 


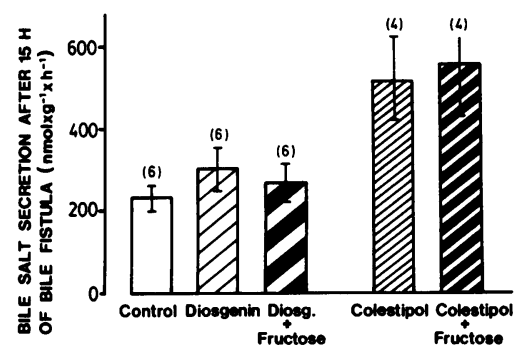

secretory function and the amount of sinusoidal free cholesterol secreted in the form of VLDL particles.

Present results are also consistent with previous studies on metabolic compartmentalization of hepatic cholesterol as related to both bile acid synthesis and biliary cholesterol secretion. Several isotopic studies have suggested that biliary cholesterol and newly synthesized bile salt molecules originate from different hepatic cholesterol precursor pools (17-23). Bile salts mainly originate from newly synthesized hepatic cholesterol (21-23) and biliary cholesterol from preformed hepatic cholesterol (20). There is also evidence suggesting that chenodeoxycholate may partially originate from a cholesterol pool different than the one from which cholic acid is formed (21). More recent studies in the acute bile fistula rat using tritiated water have demonstrated that the contribution of newly synthesized hepatic cholesterol to chenodeoxycholate, muricholate, and cholate synthesis is markedly different (50). These observations strongly suggest the existence of different subcompartments of hepatic cholesterol from which specific newly synthesized bile acids originate. Isotopic studies in man have demonstrated the presence of different hepatic precursor pools for both biliary cholesterol and newly synthesized bile salts (18). Present data are consistent with the hypothesis that the metabolically active hepatic free cholesterol used as precursor for bile salt synthesis is functionally unconnected with the compartment of free cholesterol used for both VLDL production and biliary cholesterol secretion. However, since only total bile salt synthesis was measured in the $15 \mathrm{~h}$-bile fistula rat (Fig. 4), the existence of a functional reciprocal relationship between the rates of biliary and lipoprotein cholesterol outputs and the synthesis of specific bile salt species cannot be ruled out.

Finally, the present study provides evidence to support the concept that metabolic compartmentalization represents a major determinant of biliary cholesterol output. Its significance is emphasized by the fact that cholesterol recruited per mole of bile salts and secreted into the bile may vary more than 20-fold in acute bile fistula animals, as shown in Table I

\begin{tabular}{|c|c|c|c|}
\hline & VLDL Chobolionol & 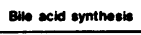 & Bubry choleeterot \\
\hline Diosgonin & $\downarrow$ & - & $T$ \\
\hline Fructione & 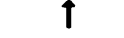 & $\overline{-}$ & $\downarrow$ \\
\hline Colbetipol & - & $T$ & - \\
\hline
\end{tabular}

Figure 5. Summary of the effects of the different diets on VLDL cholesterol, bile acid synthesis, and biliary cholesterol. (groups $B$ and $C$ ), under experimental conditions that maintain serum and hepatic cholesterol concentrations within the normal range. The cholesterol compartment supplying biliary secretion is independent of large fluctuations in cholesterol synthesis and lipoprotein uptake $(11,20)$. The excessive biliary cholesterol secretion seen in obesity (4), progestin and contraceptive steroid mixtures (51), and also in a significant proportion of patients with gallstones (1-3), is apparently determined by the amount of free cholesterol in some specific cellular subcompartment available for recruitment by the bile salts. This availability may be primarily dependent on anabolic processes, such as hepatic cholesterol esterification and VLDL production, rather than on any fundamental difference in the rates of hepatic lipoprotein cholesterol uptake or hepatic cholesterol synthesis.

\section{Acknowledgments}

The authors wish to thank the excellent technical assistance of Maria Teresa Valdivia, Maritza Iturra, and the help of Miriem Aguad in the preparation of the manuscript. They also thank Professor Christian Barth from the Institut Für Physiologie und Biochemie Der Ernährung for helpful discussion of the data.

This study was supported by a grant from the Volkswagenwerk Stiftung, grant 91/86 from the Dirección de Investigación de la Pontificia Universidad Católica de Chile, and grant 0835 from the Fondo Nacional de Desarrollo Científico y Tecnológico, Chile.

\section{References}

1. Holzbach, R. T. 1983. Pathogenesis and medical treatment of gallstones. In Gastrointestinal Disease: Pathophysiology, Diagnosis, Management. 4th ed. M. H. Sleisenger and J. S. Fordtran, editors. W. B. Saunders Co., Philadelphia. Chapter 82. 1356-1373.

2. Valdivieso, V. D., R. Palma, F. Nervi, C. Covarrubias, C. Severin, and C. Antezana. 1979. Secretion of biliary lipids in young Chilean women with cholesterol gallstones. Gut 20:997-1000.

3. Nilsell, K., B. Angelin, L. Liljeqvist, and K. Einarsson. 1985. Biliary lipid output and bile acid kinetics in cholesterol gallstone disease. Evidence for an increased secretion of cholesterol in Swedish patients. Gastroenterology. 89:287-293.

4. Bennion, L. J., and S. M. Grundy. 1975. Effects of obesity and caloric intake on biliary lipid metabolism in man. J. Clin. Invest. 56:996-1011.

5. Wheeler, H. O., and K. K. King. 1972. Biliary excretion of lecithin and cholesterol in the dog. J. Clin. Invest. 51:1337-1350.

6. Hardison, W. G. M., and J. T. Apter. 1972. Miceller theory of biliary cholesterol excretion. Am. J. Physiol. 222:61-67.

7. Wagner, C. I., B. W. Trotman, and R. D. Soloway. 1976. Kinetic analysis of biliary lipid excretion in man and dog. J. Clin. Invest. 57:473-477.

8. Salen, G., G. Nicolau, S. Shafer, and E. H. Mosbach. 1975. Hepatic cholesterol metabolism in patients with gallstones. Gastroenterology. 69:676-684.

9. Coyne, M. J., G. G. Bonoris, S. I. Goldstein, and L. J. Schoenfield. 1976. Effect of chenodeoxycholic acid and phenobarbital on the rate-limiting enzymes of hepatic cholesterol and bile acid synthesis in patients with gallstones. J. Lab. Clin. Med. 87:281-291.

10. Nervi, F. O., C. F. Covarrubias, V. D. Valdivieso, B. O. Ronco, A. Solari, and J. Tocornal. 1981. Hepatic cholesterogenesis in Chileans with cholesterol gallstone disease. Evidence for sex differences in the regulation of hepatic cholesterol metabolism. Gastroenterology. 80:539-545.

11. Turley, S. D., and J. M. Dietschy. 1979. Regulation of biliary cholesterol output in the rat: dissociation from the rate of hepatic cholesterol synthesis, the size of the hepatic cholesteryl ester pool, and 
the hepatic uptake of chylomicron cholesterol. J. Lipid Res. 20:923934.

12. Nervi, F. O., Del Pozo, C. F. Covarrubias, and B. O. Ronco. 1983. The effect of progesterone on the regulatory mechanisms of biliary cholesterol secretion in the rat. Hepatology. 3:360-367.

13. Del Pozo, R., F. Nervi, C. Covarrubias, and B. Ronco. 1983. Reversal of progesterone-induced biliary cholesterol output by dietary cholesterol and ethynylestradiol. Biochim. Biophys. Acta. 753:164172.

14. Nervi, F., M. Bronfman, W. Allalón, E. Depièreux, and R. Del Pozo. 1984. Regulation of biliary cholesterol secretion in the rat. Role of hepatic cholesterol esterification. J. Clin. Invest. 74:2226-2237.

15. Stone, B. G., S. K. Erickson, W. Y. Craig, and A. D. Cooper. 1985. Regulation of rat biliary cholesterol secretion by agents that alter intrahepatic cholesterol metabolism. J. Clin. Invest. 76:1773-1781.

16. Drevon, G. A., S. C. Engelhorn, and D. Steinberg. 1980. Secretion of very low density lipoproteins enriched in cholesteryl esters by cultured rat hepatocytes during stimulation of intracellular cholesterol esterification. J. Lipid Res. 21:1065-1071.

17. Schwartz, C. C., Z. R. Vlahcevic, L. C. Halloran, D. H. Gregory, J. B. Meck, and L. Swell. 1975. Evidence for the existence of definitive hepatic cholesterol precursor compartments for bile acids and biliary cholesterol in man. Gastroenterology. 69:1379-1382.

18. Schwartz, C. C., M. Berman, Z. R. Vlahcevic, L. Gregg Halloran, D. H. Gregory, and L. Swell. 1977. Multicompartmental analysis of cholesterol metabolism in man. Characterization of the hepatic bile acid and biliary cholesterol precursor sites. J. Clin. Invest. 61:408-423.

19. Staple, E., and S. Gurin. 1954. The incorporation of radioactive acetate into biliary cholesterol and cholic acid. Biochim. Biophys. Acta. 15:372-376.

20. Turley, S. D., and J. M. Dietschy. 1982. Cholesterol metabolism and excretion. In The Liver: Biology and Pathobiology. I. Arias, H. Popper, D. Schachter, and D. A. Shafritz, editors. Raven Press, New York. 467-492.

21. Mitropoulos, K. A., N. B. Myant, G. F. Gibbons, S. Balasubramanian, and B. E. A. Reeves. 1974. Cholesterol precursor pools for the synthesis of cholic and chenodeoxycholic acids in rats. J. Biol. Chem. 249:6052-6056.

22. Cronholm, T., A. L. Burlingame, and J. Sjöval. 1974. Utilization of the carbon and hydrogen atoms of ethanol in the biosynthesis of steroids and bile acids. Eur. J. Biochem. 49:497-510.

23. Normann, P. T., and K. R. Norum. 1974. Newly synthesized hepatic cholesterol as precursor for cholesterol and bile acids in rat bile. Scand. J. Gastroenterol. 11:427-432.

24. Schwartz, C. C., L. G. Halloran, Z. R. Vlacevic, D. H. Gregory, and L. Swell. 1978. Preferential utilization of free cholesterol from high-density lipoproteins for biliary cholesterol secretion in man. Science (Wash. DC). 200:62-64.

25. Topping, D. L., and P. A. Mayes. 1972. The immediate effects of insulin and fructose on the metabolism by the perfused liver. Biochem. J. 126:295-311.

26. Parkinson, T. M., K. Gunderson, and N. A. Nelson. 1970. Effects of colestipol (U-26, $597 \mathrm{~A}$ ), a new bile-sequestrant, on serum lipids in experimental animals and man. Atherosclerosis. 11:531-537.

27. Myant, N. B., and H. A. Eder. 1961. The effect of biliary drainage upon the synthesis of cholesterol in the liver. J. Lipid Res. 2:363-368.

28. Krebs, H. A., and K. Henseleit. 1932. Untersuchungen über die Harnstoffbildung im Tierkörper. Hoppe-Seyler's Z. Physiol. Chem. 210:33-66.

29. Hamilton, R. L., M. N. Berry, M. C. Williams, and E. M. Severinghaus. 1974. A simple and inexpensive membrane "lung" for small organ perfusion. J. Lipid Res. 15:182-186.

30. Berry, M. N., and D. S. Friend. 1969. High-yield preparation of isolated rat liver parenchymal cells. A biochemical and fine structural study. J. Cell Biol. 43:506-520.
31. Frantz, I. D., Jr., E. Dulit, and A. C. Davidson. 1957. The state of esterification of the sterols of rat skin. J. Biol. Chem. 206:139-144.

32. Zak, B. 1957. Simple rapid microtechnic for serum total cholesterol. Am. J. Clin. Pathol. 27:583-588.

33. Baginski, E. S., P. P. Fos, and B. Zak. 1967. Microdetermination of inorganic phosphate, phospholipids and total phosphate in biological material. Clin. Chem. 13:326-332.

34. Talalay, P. 1960. Enzymic analysis of steroid hormones. Methods Biochem. Anal. 8:119-143.

35. Turley, S. D., and J. M. Dietschy. 1978. Reevaluation of the 3 $\alpha$-hydroxysteroid dehydrogenase assay for total bile acids in bile. $J$. Lipid Res. 19:924-928.

36. Lowry, O. H., N. J. Rosebrough, A. L. Farr, and Q. J. Randall. 1951. Protein measurement with the Folin phenol reagent. J. Biol. Chem. 193:265-275.

37. Snedecor, G. W., and W. G. Cochran. 1967. Statistical Methods. 6th ed. Iowa State University Press, Ames, IA.

38. Percy-Robb, I. W., and G. S. Boyd. 1970. The synthesis of bile acids in perfused rat liver subjected to chronic biliary drainage. Bio chem. J. 118:519-530.

39. Noel, S. P., L. Wong, P. J. Dolphin, L. Dory, and D. Rubinstein. 1979. Secretion of cholesterol-rich lipoproteins by perfused livers of hypercholesterolemic rats. J. Clin. Invest. 64:674-683.

40. Ide, T., and J. A. Ontko. 1981. Increased secretion of very low density lipoprotein triglyceride following inhibition of long chain fatty acid oxidation in isolated rat liver. J. Biol. Chem. 256:10247-10255.

41. Gregory, D. H., Z. R. Vlahcevic, P. Schatzki, and L. Swell. 1975. Mechanism of secretion of biliary lipids. I. Role of bile cannalicular and microsomal membranes in the synthesis and transport of biliary lecithin and cholesterol. J. Clin. Invest. 55:105-114.

42. Marsh, J. B. 1974. Lipoproteins in a nonrecirculating perfusate of rat liver. J. Lipid Res. 15:544-550.

43. Lange, Y., and T. L. Steck. 1985. Cholesterol-rich intracellular membranes: A precursor to the plasma membrane. J. Biol. Chem. 29:15592-15597.

44. Robins, S. J., and H. Brunengraber. 1982. Origin of biliary cholesterol and lecithin in the rat: contribution of new synthesis and preformed hepatic stores. J. Lipid Res. 23:604-608.

45. Mitropoulos, K. A., S. Venkatesan, S. Balasubramaniam, and T. J. Peters. 1978. The submicrosomal localization of acyl-coenzyme A-cholesterol acyltransferase and its substrate, and of cholesteryl esters in rat liver. Biochem. J. 174:836-872.

46. Ulloa, N., J. Garrido, and F. Nervi. 1987. Ultracentrifugal isolation of vesicular carriers of biliary cholesterol in native human and rat bile. Hepatology. 7:235-244.

47. Hamilton, R. L., D. M. Regen, M. E. Gray, and V. S. Lequire. 1967. Lipid transport in rat liver. 1. Electron microscopic identification of very low density lipoproteins in perfused rat liver. Lab. Invest. 16:305-319.

48. Alexander, C. A., R. L. Hamilton, and R. Havel. 1976. Subcellular localization of B apoprotein of plasma lipoproteins in rat liver. $J$. Cell Biol. 69:241-263.

49. Gregory, D. H., Z. R. Vlahcevie, M. F. Prugh, and L. Swell. 1978. Mechanism of secretion of biliary lipids: role of a microtubular system in hepatocellular transport of biliary lipids in the rat. Gastroenterology. 74:93-100.

50. Stange, E. F., D. K. Spady, and J. M. Dietschy. 1985. Characterization of the cholesterol precursor pools used for bile acid synthesis in the rat. In Enterohepatic Circulation of Bile Acids and Sterol Metabolism. G. Paumgartner, G. Gerok, and A. Stihl, editors. M.T.P. Press Ltd., Lancaster, England. 29-36.

51. Kern, F., G. T. Everson, B. De Mark, C. Mekinley, R. Sholwalter, D. Z. Braverman, P. Szczepanik-Van Leeuwen, and P. D. Klein. 1982. Biliary lipids, bile acids, and gallbladder function in the human female: effects of contraceptive steroids. J. Lab. Clin. Med. 99:798-805. 